\title{
Vom Sehen zum Lesen. \\ Eine Fallstudie zur ikonologischen Praxis der Nachkriegszeit
}

\author{
Ulrich Rehm
}

Im Jahr 1956 trat ein 32jähriger Kunsthistoriker gleich mit mehreren Beiträgen in westdeutschen Fachzeitschriften zur Kunstgeschichte hervor. Willibald Sauerländer (geb. 1924) publizierte damals neben einem kleineren Text in der Kunstchronik ${ }^{1}$ zwei umfangreichere Aufsätze zu ganz unterschiedlichen Themen: in der Zeitschrift für Kunstgeschichte »Beiträge zur Geschichte der sfrühgotischen $<$ Skulptur $\ll,{ }^{2}$ im Münchner Jahrbuch der Bildenden Kunst »Die Jahreszeiten. Ein Beitrag zur allegorischen Landschaft beim späten Poussin ${ }^{3}{ }^{3}$

Nicht nur in der Wahl der Themen, auch in der des Vokabulars ist der Kontrast zwischen den zwei Aufsätzen beträchtlich. Und ganz offensichtlich geht das mit einem Wechsel der methodischen Perspektive einher. Auch wenn dieser Wechsel eng mit der individuellen Biographie verknüpft ist und in den Arbeiten Sauerländers selbst keine unmittelbare Fortsetzung findet, so ist er doch signifikant für bestimmte Entwicklungen der Kunstgeschichte im westlichen Nachkriegsdeutschland, die allerdings erst mit erheblichen Verzögerungen an Profil gewinnen. Von besonderem Interesse ist, daß das gleichzeitige Auftreten zweier ganz unterschiedllicher Perspektiven im Jahr 1956 das Resultat einer radikalen Distanzierung von der Nachkriegskunstgeschichte in Westdeutschland war, genauer gesagt: vom Münchener Kunsthistorischen Institut.

1953 hatte Sauerländer, 29jährig, bei Hans Jantzen in München mit einer Arbeit über "Das gotische Figurenportal in Frankreich. Studien zur Geschichte der französischen Portalskulptur von Chartres West bis zum Reimser Josephsmeister" promoviert. Im

1 Willibald Sauerländer, Zu den Westportalen von Chartres, in: Kunstchronik 9, 1956, $155 f$.

2 Beiträge zur Geschichte der sfühgotischen<Skulptur, in: Zeitschrift für Kunstgeschichte 19, 1956, $1-33$.

3 Die Jahreszeiten. Ein Beitrag zur allegorischen Landschaft beim späten Poussin, in: Münchner Jahrbuch der Bildenden VII, 1956, 1-33; wiederabgedruckt in: Willibald Sauerländer, Geschichte der Kunst - Gegenwart der Kritik, hg. von Werner Busch, Wolfgang Kemp, Monika Steinhauser und Martin Warnke, Köln 1999, 90-116. 
darauffolgenden Jahr hatte er eine Bildmonographie zur Kathedrale von Chartres herausgegeben und eingeleitet. ${ }^{4}$

Sauerländer selbst charakterisiert seine 1946/47 begonnenen Münchener Studienjahre als von einer gewissen Provinzialität geprägt. ${ }^{5}$ Allein der ehemalige Wölfflin-Assistent Franz Roh habe sich für die Moderne engagiert und das Gespräch als Unterrichtsform gesucht. ${ }^{6}$ Im übrigen habe sich unter dem Einfluß Jantzens eine ablehnende Haltung gegenüber bestimmten Formen des Intellektualismus etabliert. Dieser sei die antiquarisch-positivistische Tradition Adolf Goldschmidts ebenso suspekt gewesen wie die ikonologische Erwin Panofskys. ${ }^{7}$ Selbst im Fall Hans Sedlmayrs, der 1951 an die Münchener Universität kam, soll sich der widerstrebende Affekt vieler Studierender, so Sauerländer später, zunächst weniger gegen dessen nationalsozialistische Vergangenheit gerichtet haben, sondern vielmehr gegen das vermeintlich »höhere $\mathrm{Maß}$ an Begriffsschärfe«, gegen den Einbruch einer gewissen intellektuellen Kälte in das »vertraute Gehege einer 'gemütlich< geschützten Kunstgeschichte «. ${ }^{8}$ Die studentischen Proteste gegen Sedlmayr hätten geradezu die »Verweigerung eines Modernisierungsschubes bekundet. ${ }^{9}$

Der Wunsch der damaligen Studierenden nach Kontinuität in den Gegenständen und Methoden war offensichtlich so groß, daß die Frage, inwieweit sich die Kunstgeschichte in Deutschland während der Zeit des Nationalsozialismus diskreditiert hatte, zunächst zweitrangig blieb.

Für Sauerländer war es der 1954 erfolgte Schritt nach Paris, mit dem er sich aus der Enge der Münchener Kunstgeschichte zu befreien versuchte. Daß er gleich fünf Jahre blieb, entsprach, nach eigenem Bekunden, dem Bedürfnis nach einer radikalen Distanzierung vom geistigen Klima in Deutschland, von dem er die deutschen Forschungsinstitute in Italien ausdrücklich nicht ausschließt. ${ }^{10}$

4 Die Kathedrale von Chartres, Stuttgart 1954.

5 Willibald Sauerländer, Zersplitterte Erinnerung, in: Kunsthistoriker in eigener Sache. Zehn autobiographische Skizzen, hg. von Marina Sitt. Mit einer Einleitung von Heinrich Dilly, Berlin 1990, 301-323, hier 311 (wiederabgedruckt: Geschichte der Kunst - Gegenwart der Kritik, hg. von Werner Busch, Wolfgang Kemp, Monika Steinhauser und Martin Warnke, Köln 1999, 7-27).

6 Sauerländer 1990 (wie Anm. 5), 308. Vgl. auch: Katrin Meier-Wohlt, Das Kunsthistorische Seminar der Universität München zur Zeit des Nationalsozialismus, in: Kunstgeschichte im Nationalsozialismus. Beiträge zur Geschichte einer Wissenschaft zwischen 1930 und 1950, Weimar 2005, 85-97; Thomas Lersch, Schreibverbot? Erkundungen zu Franz Roh, ebd., 161-181.

7 Sauerländer 1990 (wie Anm. 5), 309f.

8 Sauerländer 1990 (wie Anm. 5), 311; Sauerländer hatte sich in einem Zeitungsartikel gleich 1951 gegen die von Sedlmayr errichteten »schillernden Heilsgebäude « gewandt: Willibald Sauerländer, Schillernde Kunsttheorie. Zu Hans Sedlmayrs neuem Buch »Die Entstehung der Kathedrale«, in: Neue Zeitung, März oder April 1951. - Vgl. auch Willibald Sauerländer, Der Münchner Protest gegen die Berufung Hans Sedlmayrs im Frühjahr 1951, in: 200 Jahre Kunstgeschichte in München. Positionen, Perspektiven, Polemik. 1780-1980, hg. von Christian Drude und Hubertus Kohle, München und Berlin 2003, 182-198, hier 189.

9 Ebd., 194.

10 Sauerländer 1990 (wie Anm. 5), 312. 
Welchen Stellenwert innerhalb dieser Entwicklung nehmen die beiden genannten Aufsätze ein, deren Erscheinen bereits in die Pariser Zeit des Autors fällt? Ich erlaube mir, diese Frage zu verfolgen, ohne näher auf die Gegenstände und deren Interpretation einzugehen, indem ich lediglich auf markante Formulierungen achte.

Auffällig am Aufsatz über die frühgotische Skulptur in Frankreich ist, daß er - noch in enger Verbundenheit zur phänomenologischen Stilanalyse - als das wesentliche Erkenntnismoment und -interesse expressis verbis das Sehen benennt. ${ }^{11}$ Das vorläufige Ziel bestehe darin, die wichtigsten erhaltenen Denkmäler zu »sichten « (kursive Hervorhebungen vom Verf.). Längerfristig gehe es darum, »die Genesis des jüngeren Chartreser und des Reimser Stils klar sehen " zu können. Auch im Detail ist der Gesichtssinn die Leitmetapher der Untersuchung: Es fällt »Nenes Licht « auf bestimmte Sachzusammenhänge, Befunde lassen sich "unschwer erkennen ", man schenkt "Aufmerksamkeit «, es "zeigt sich überraschende Verwandtschaft«. Um eine "Annahme zu prüfen «, müssen die Dinge "genauer angeseben werden«. Man muß die Objekte nur gegeneinander halten, »um zu seben ... «. Was auf Abbildungen »nie erkennbar « ist, ist »im Original aber leicht zu sehen «. Und das eine oder andere schließlich »bleibt in Betracht zu ziehen «.

Die einfühlsamen Beschreibungen der Kunstobjekte treten im Text an die Stelle der Skulpturen selbst, und in den sprachlichen Übereinstimmungen oder Differenzen der Charakterisierungen erweist sich der Grad an Verwandschaft unter dem Leitgedanken einer konsequenten Stilentwicklung.

Schon bei Sauerländers erster Begegnung mit Louis Grodecki 1954, also zwei Jahre vor Erscheinen des Skulpturenaufsatzes, in Paris soll dieser die Vorstellung gesetzmäßiger Stiletwicklung harsch zurückgewiesen haben, ${ }^{12}$ die deutlich in der Tradition Jantzens steht. ${ }^{13}$ War nach Sauerländers eigenem Bekunden damit in München eine »ehrfurchtgebietende Auratisierung « der Kunstwerke verbunden, so ist in der betont klaren und scharfsinnigen Diktion seines eigenen Textes davon wenig zu bemerken. ${ }^{14}$

Während im Aufsatz über die frühgotische Skulptur also der wesentliche Akzent auf dem Sehen liegt, so ist das leitende Konzept im Aufsatz über die "Jahreszeiten« Poussins das Lesen.

Bevor es an die eigentlichen Fragestellung geht, werden zunächst Texte von Félibien, Le Brun, Diderot und Constable referiert. Der maßgebliche Anspruch der Bildlektüre, die dann folgt, ist weniger der, die stilistischen Eigenschaften oder inhaltlichen Aussagen der Bilder möglichst unmittelbar in Sprache zu übertragen. Zwar betonen auch hier Begriffe wie »genaueres Zusehen « die Wichtigkeit der sinnlichen Tätigkeit. Insgesamt zielt der Aufsatz jedoch von vornherein auf das Erschließen einer das »Ganze tragenden Bildidee«. Der Lektüreakt richtet sich also auf etwas nicht unmittelbar Sichtbares.

11 Sauerländer 1956 (Beiträge; wie Anm. 2).

12 Sauerländer 1990 (wie Anm. 5), 311f.

13 Vgl. Sauerländer 1990 (wie Anm. 5), 310: »Im Kreis der Jantzenschüler wurde sSehen zu einer Art nicht mehr rationaler, auch nicht mehr lernbarer Erfahrung, zu einer Erleuchtung, die mysteriösen Zugang zum Wesen des Kunstwerks zu gewähren schien«.

14 Sauerländer 1990 (wie Anm. 5), 315. 
Sauerländer deutet die Jahreszeiten allegorisch, und zwar als Repräsentanten verschiedener Zeitalter der christlichen Heilsgeschichte. Dabei wird den »kleinen Figuren « im Bild, das heißt den Trägern der dargestellten alttestamentlichen Handlungen, eine »eindringliche Sprachgewalt« zugesprochen. ${ }^{15}$

$\mathrm{Da}$ ist vom "Schlüssel zum eigentlich idealen Thema « die Rede, vom »Entziffern « oder »Typologisch-Lesen «, vom »Herausschälen«, von »Gedanken hinter dem Bild «, von »verborgenen « oder »fernen Hinweisen«. Und schließlich heißt es: »Erst die Kenntnis von dieser Auslegung erläutert, warum Poussin der Begegnung von Boas und Ruth, von Sponsus und Sponsa, so feierlichen Charakter verlieh «. ${ }^{16}$

Der Autor präsentiert sich so als jemand, der aufgrund seiner Belesenheit den Schlüssel zur eigentlichen, verborgenen Bedeutung des Kunstwerks besitzt und damit das Ideale oder Wahre hinter dem Bild zu eröffnen vermag. Gerade in diesem Punkt darf man auf die erst jünst erfolgte Revision Sauerländers gespannt sein, mit deren Publikation demnächst zu rechnen ist.

Während die einfühlende Beschreibung der Kunstwerke im Laufe der 50er Jahre offenbar begann, dem Verdacht der Subjektivität (und damit der Verführbarkeit) zu unterliegen, erlaubte es die in der Nachkriegszeit zunehmend praktizierte Ausrichtung der Ikonologie, das dargestellte Sujet als »Hülle« zu begreifen, in der »eine höhere, ideale Welt zur Anschauung kommt " ${ }^{17}$ Das heißt, an die Stelle der Einfühlung in die Körperlichkeit des Kunstwerks mit Hilfe der Sinne, trat eine Entkörperlichung der Kunst zugunsten einer Auffassung der Symbolhaftigkeit des Dargestellten, die intellektuell zu erfassen und durch Textquellen zu bestätigen sei. Damit verwandelte sich die Rolle der Kunsthistoriker von der einfühlsamer Seher und Übersetzer in die intellektueller Leser und Ausleger. An die Stelle der Sinnlichkeit trat der Intellekt, der zugleich eine Idealisierung der Gegenstände erlaubte.

Unter diesem Gesichtspunkt ist die Wendung von der Skulptur zur Malerei auffällig: War die Skulptur das ideale Erprobungsfeld der körperlichen Einfühlung gewesen, so begann nunmehr - wenn auch langsam - die Malerei zum Hauptgegenstand allegorischer Lektüre zu avancieren, deren Wirkungsfeld man in einer Tiefenschicht hinter der eigentlichen Gemäldeoberfläche lokalisierte. Auch im Jahreszeiten-Aufsatz heißt es ausdrücklich: »Was aber eigentlich mit dem Bilde gesagt werden soll, liegt überhaupt jenseits der in der sinnlichen Wahrnehmung sofort erschaubaren Schicht ${ }^{18}{ }^{18}$

Die hier zum Tragen kommende Auffassung läßt sich im weitesten Sinne als ikonologische bezeichnen - allerdings in einer sehr speziellen Ausrichtung und noch weitgehend unbeschwert von den methodologischen Überlegungen, wie Panofsky sie schon in den 1930er Jahren angestellt hatte. Sauerländer charakterisierte diese Ausrichtung selbst folgendermaßen: „Überall entdeckte man jetzt verborgene Bedeutungen. >Disguised

15 Sauerländer 1956 (Jahreszeiten; wie Anm. 3), 173.

16 Ebd., 175.

17 Ebd., 172.

18 Ebd., 174. 
Symbolism<, gestern noch verdammt, hatte nun Konjunktur. «19 Tatsächlich hatte Panofsky das Konzept des »disguised symbolism«, wenn auch etwa zeitgleich, unabhängig von seinem Ikonologie-Modell entwickelt. Und er wollte die Gültigkeit dieses »disguised symbolism« auf ganz spezifische Phänomene der altniederländischen Malerei beschränkt wissen. In seinem ersten regelrechten Exiljahr 1934 hatte er dies in seinem Aufsatz über Jan van Eycks Arnolfini-Portrait im Burlington Magazine publik gemacht. ${ }^{20}$

Das Ikonologie-Modell, sprich: der Entwurf einer ikonologischen Methode, wurde kaum unmittelbar angewandt oder diskutiert. Der inzwischen zum kunsthistorischen Standardtext avancierte Aufsatz »Zum Problem der Beschreibung und Inhaltsdeutung von Werken der bildenden Kunst« von 1932 mit dem berühmten ikonologischen Dreistufenmodell in seiner ersten Formulierung war weitgehend vergessen. ${ }^{21}$ Bekannter dürften die 1939 erschienenen »Studies in Iconology " gewesen sein, in deren Einleitung Panofsky seine Überlegungen zu einem Dreistufenmodell von 1932 erneut aufgegriffen hatte, wenn auch erheblich modifiziert. ${ }^{22}$ Sauerländer bekundet, er selbst habe die "Studies « erst 1954 in Paris gelesen. ${ }^{23}$ Doch in der Praxis kam es kaum zu einer ernsthaften Auseinandersetzung mit den Ikonologie-Modellen.

Nicht daß Panofsky im Nachkriegsdeutschland nicht zitiert worden wäre. In seinem Skulpturenaufsatz diskutiert auch Sauerländer ganz selbstverständlich einen Datierungsvorschlag Panofskys. ${ }^{24}$ Doch gerade die methodologisch orientierten Arbeiten waren offensichtlich dem Phänomen »binnendeutscher Verdrängungen « zum Opfer gefallen, und das blieb zunächst auf weite Strecken auch so. ${ }^{25}$

Im Jahreszeiten-Aufsatz fällt der Name Panofskys nicht. Trotzdem gehörte er zu den Adressaten dieses Textes: Sauerländer sandte ihm einen Sonderdruck in die USA, verbunden mit dem entschuldigenden Hinweis, es handle sich um den Versuch eines Anfängers. ${ }^{26}$ Die Bedeutung dieser Kontaktaufnahme eines jungen westdeutschen Nachkriegs-Kunsthistorikers mit einem exilierten Fachvertreter ist nicht zu unterschätzen: Er darf zu den wenigen geglückten Versuchen zählen, den Dialog mit einem exilierten Kollegen - auch auf Dauer - aufzunehmen.

In der Nachkriegszeit fungierte wohl schon das bloße Bewußtsein für die Existens einer ikonologischen Methode als wissenschaftlicher Garant für das grundsätzliche Vor-

19 Sauerländer 1990 (wie Anm. 5), 315.

20 Jan van Eyck's Arnolfini Portrait, in: The Burlington Magazine 64, 1934, 117-127.

21 Erwin Panofsky, Zum Problem der Beschreibung und Inhaltsdeutung von Werken der bildenden Kunst, in: Logos 21, 1932, 103-119, hier zitiert: Neudruck in: Ekkehard Kaemmerling (Hg.), Bildende Kunst als Zeichensystem Band 1, Ikonographie und Ikonologie. Theorien - Entwicklung Probleme (DuMont-Taschenbücher, Bd. 83), Köln 1979, 185-206.

22 Erwin Panofsky, Studies in Iconology. Humanistic Themes In the Art of the Renaissance, New York 1939, hier besonders: Introductory, 3-31.

23 Sauerländer 1990 (wie Anm. 5), 315.

24 Sauerländer 1956 (Beiträge; wie Anm. 2), 8.

25 Sauerländer 1990 (wie Anm. 5), 314.

26 Ebd. 
handensein versteckter Symbolik in der bildenden Kunst. "Disguised symbolism« avancierte zum Synonym von Ikonologie. Ein erster Höhepunkt dieser Konjunktur mag erreicht gewesen sein, als Walter Friedländer mit Bezug auf Sauerländers PoussinAufsatz die »Vier Jahreszeiten « als den Gipfel versteckter Symbolik pries: Die Bedeutung der vier Gemälde Poussins, so schrieb er in seiner Monographie von 1966, sei so tief versteckt, daß sie lange Zeit als bloße Darstellung der wechselnden Jahreszeiten aufgefaßt worden seien. ${ }^{27}$

1956, also im Jahr des Aufsatz-Debuts Sauerländers, erlangte das Konzept versteckter Symbolik neue Aktualität: Otto Pächt (1902-1988) reagierte mit deutlich anti-rationalistischem Affekt im Burlington Magazine mit zum Teil heftiger Kritik auf Panofskys drei Jahre zuvor erschienene »Early Netherlandish Paintings «, deren Argumentation das Konzept des »disguised symbolism « weiter ausführt. ${ }^{28}$ In dieser Auseinandersetzung zwischen zwei Exilanten, ${ }^{29}$ kritisierte Pächt aus einer der Sedlmayrschen Strukturanalyse nahestehenden Position heraus einen Ansatz, der zu diesem Zeitpunkt in Deutschland gerade begann, mit dem Anspruch eines methodischen Neuaufbruchs in Erscheinung zu treten.

Aus heutiger Perspektive betrachtet, verband sich dieser Neuaufbruch in Deutschland jedoch zunächst, wie Sauerländer im Rückblick einräumt, mit dem Wunsch, den » Ängsten vor Veränderungen und Umstürzen « der Nachkriegszeit zu begegnen: ${ }^{30}$ »disguised symbolism « war, seines ursprünglichen Kontextes beraubt, das Rezept, mit dem man die Entwicklungen der jüngeren Kunstgeschichte als Konsequenz einer bis in das Mittelalter zurückreichenden Tradition darstellen konnte.

Im Nachkriegsdeutschland prägte nunmehr das christliche Mittelalter als der paradiesische Fluchtpunkt der Weltgeschichte die ikonologischen Untersuchungen. War während der Zeit des Nationalsozialismus die kunsthistorische Beschäftigung mit mittelalterlichen Themen im Extremfall bis zum Schwelgen in einer Art Urgermanentum mutiert, so folgte nunmehr die Projektion der gesamteuropäischen Kultur auf eine vermeintliche gemeinsame Wiege im christlichen Mittelalter - eine Projektion, die Sauerländer selbst 1992 in seinem Aufsatz "Von den sonderleistungen Deutscher Kunst zur >Ars Sacra< - Kunstgeschichte in Deutschland 1945-1950 « scharf brandmarkte. ${ }^{31}$

Im Jahreszeiten-Aufsatz von 1956 griff Sauerländer, dem die Quellen des Mittelalters besonders nahe lagen, selbst vielfach auf mittelalterliches Text- und Bildmaterial zurück

27 Walter Friedländer, Nicolas Poussin. A New Approach, London 1966, 193.

28 Otto Pächt, The Burlington Magazine 98, 1956, 110-116 und 267-279; vgl, auch ders., Methodisches zur kunsthistorischen Praxis. Ausgewählte Schriften, hg. von J. Oberhaidacher, Arthur Rosenauer und G. Schikola, München 1977, 235-250 und 314-315.

29 Otto Pächt verließ sein englisches Exil erst 1963 und hielt sich 1956 gerade als Fellow am Institute for Advanced Study in Princeton auf.

30 Sauerländer 1990 (wie Anm. 5), 315.

31 In: Wissenschaft im geteilten Deutschland. Restauration oder Neubeginn nach 1945, hg. von Walter H. Pehle und Peter Sillem, Frankfurt a. M. 1992, 177-190, 244f.; wiederabgedruckt in: Willibald Sauerländer, Geschichte der Kunst - Gegenwart der Kritik, hg. von Werner Busch, Wolfgang Kemp, Monika Steinhauser und Martin Warnke, Köln 1999, 277-292. 
und machte in seiner Interpretation ausgiebig vom Prinzip der Typologie Gebrauch, deren Anwendung im Mittelalter weit selbstverständlicher war als in weiten Phasen der frühen Neuzeit. Damit geriet der Aufsatz - zumindest dem Anschein nach - partiell in die Nähe zu jener Tendenz, die Sauerländer selbst immer wieder ganz entschieden abgelehnt hat.

Es könnte sogar der Eindruck entstehen, Sauerländers Interpretationsansatz weise gewisse Parallelen zu Hans Sedlmayrs Interpretation von Pieter Brueghels »Blindensturz« auf. Diese hatte Sedlmayr 1951 bereits als Münchener Antrittsvorlesung einem großen Publikum vorgetragen und ein Jahr nach Sauerländers Jahreszeiten-Aufsatz publiziert. Anders als Sauerländer trat Sedlmayr dabei mit einem explizit methodologischen Anspruch auf. Dessen Kern besteht in einer durchaus fragwürdigen Übertragung mittelalterlicher Auslegungsmethode auf ein neuzeitliches Kunstwerk, die zudem noch von einem stupiden Schematismus ist: Die verschiedenen Ebenen mittelalterlicher Bibelauslegung sollten auf das Bild angewandt werden. ${ }^{32}$

Was die beiden Aufsätze Sauerländers und Sedlmayrs miteinander verbindet, ist die heilsgeschichtliche Ausrichtung, die zudem in beiden Fällen in der Aussage »extra ecclesiam nulla salus « - außerhalb der Kirche kein Heil - gipfelt. ${ }^{33}$ Für Sauerländer war dies eine inhaltliche Komponente des Sintflut-Gemäldes; für Sedlmayr hingegen ein persönliches weltanschauliches Credo. Was Sauerländer von diesem hielt, soll er Sedlmayr unmittelbar nach dessen Antrittsvorlesung ins Gesicht gesagt haben: »Herr Professor, wir sind die Blinden, die nicht zur Kirche kommen und auch nie zur Kirche kommen werden «. ${ }^{34} \mathrm{Da}$ Sauerländers Jahreszeiten-Aufsatz nicht explizit eine weltanschauliche Position formuliert, war er offenbar leicht mißzuverstehen.

Es verwundert daher nicht allzu sehr, daß der 1952 aus dem Exil heimgekehrte Kurt Badt, der 1961 bereits auf Sedlmayrs Vermeer-Interpretation ${ }^{35}$ mit einer Streitschrift reagiert hatte, ${ }^{36}$ in seiner Poussin-Monographie von 1969 die Thesen Sauerländers heftig attackierte. ${ }^{37}$ Bezeichnenderweise wandte Badt sich genau gegen die Vorstellung von einem »christlich-katholischen « Poussin und versuchte stattdessen, das "pagan-antikische « Weltverständnis des Malers nachzuweisen - und dies gerade nicht durch ikonologische Argumentation, deren scharfe Ablehnung unübersehbar ist, sondern aus einer

32 Hans Sedlmayr, Pieter Bruegel. Der Sturz der Blinden. Paradigma einer Strukturanalyse, in: Hefte des Kunsthistorischen Instituts der Universität München 2, 1957, 1-35.

33 Sauerländer 1956 (Jahreszeiten; wie Anm. 3), 183; Sauerländer 2003 (wie Anm. 8), $191 \mathrm{f}$.

34 Ebd., 192.

35 Hans Sedlmayr, Jan Vermeer: Der Ruhm der Malkunst, in: Festschrift für Hans Jantzen, Berlin 1951, 169-177.

36 Kurt Badt, Modell und Maler. Probleme der Interpretation. Eine Streitschrift gegen Hans Sedlmayr, Köln 1961, Ndr. 1997; vgl. auch: Hans Sedlmayr, Jan Vermeer. De schilderkunst. Nach Badt zugleich Replik, in: Hefte des Kunsthistorischen Seminars der Universität München 7-8, 1962; dazu: Lida von Mengden, Vermeers >De Schilderkonst، in den Interpretationen von Kurt Badt und Hans Sedlmayr, Frankfurt a. M., Bern, New York 1984.

37 Kurt Badt, Die Kunst des Nicolas Poussin, Bd. 1-2, Köln 1969, hier Textbd., 556-568. 
Art hermeneutischer Analyse des Gesamtwerks Poussins heraus, die - zumindest in diesem speziellen Fall - nicht gerade überzeugt.

Die Rückführung neuzeitlicher oder moderner Darstellungen auf mittelalterliche Traditionen, wie sie seit den 50er Jahren vielfach praktiziert wurde, ist jedoch alles andere als eine zwingende Folgerung aus der ikonologischen Methode. In den ikonologischen Schlüsseltexten Aby Warburgs über das Bildprogramm des Palazzo Schifanoia (1912) ${ }^{38}$ sowie Erwin Panofskys und Fritz Saxls über »Classical Mythology in Medieval Art « $(1933)^{39}$ war vielmehr ein ganz anderes, hintergründiges Entwicklungsmodell zum Tragen gekommen: Hier wird unterstellt, der vitale Kern der klassisch-antiken Kunst habe mit der Wanderung der Bildmotive in komplexen Assimilierungsprozessen die relative Ignoranz des Mittelalters überlebt, um in der Renaissance in ihrer ursprünglichen Gestalt wieder in Erscheinung zu treten.$^{40}$ Es wird also gerade der Aspekt der Zäsur zwischen Mittelalter und früher Neuzeit betont.

Als Sauerländer seinen Poussin-Aufsatz im Erscheinungsjahr Erwin Panofsky zusandte, verband er dies mit der besagten Bitte um Entschuldigung seiner Anfängerschaft und bewertete diesen Sachverhalt später als »von allgemeinerer wissenschaftsgeschichtlicher Bedeutung «. ${ }^{41}$ Gerade vor dem Hintergrund der Münchener Entwicklungen muß die Aneignung einer ikonologischen Perspektive tatsächlich als eine Art erneuten Gehenlernens empfunden worden sein, wohl verbunden mit dem Wunsch, sich eine methodische Basis anzueignen, die den Münchener `Sumpf< aufmischen und zudem beanspruchen konnte, intellektueller Überprüfung standzuhalten. Während Form- und Stilgeschichte vielfach dem ideologischen Mißbrauch verfallen gewesen waren oder zumindest als Refugium des politischen Überwinterns hatten genutzt werden können, gelangte die Ikonologie in Deutschland nach und nach in den Ruf eines vom Dritten Reich verhinderten wissenschaftlichen Fortschritts. ${ }^{42}$ Zwar war auch sie mit Fachvertretern wie Hans Kauffmann während des Nationalsozialismus nicht ganz untergegangen ${ }^{43}$ hatte aber, aufs Ganze gesehen, doch ein Nischendasein geführt.

Daß Panofsky seine Ikonologie-Modelle ursprünglich auf das eingeschränkte Phänomen der Beschreibung und Inhaltsdeutung hin konzipiert hatte, lag damals ebenso außerhalb des allgemeinen Bewußtseins wie die Tatsache, daß es wesentliche Wurzeln in

38 Aby Warburg, Italienische Kunst und internationale Astrologie im Palazzo Schifanoja zu Ferrara (1912), in: Aby Warburg, Gesammelte Schriften, hg. von Gertrud Bing, Leipzig und Berlin 1932, Bd. 2, 459-481.

39 Erwin Panofsky und Fritz Saxl, Classical Mythology in Mediaeval Art, in: Metropolitan Museum Studies, IV, 2, 1933, 228-280.

40 Auch dieses Modell unter zeitgeschichtlichen Gesichtspunkten zu untersuchen, erscheint mir außerordentlich vielversprechend.

41 Sauerländer 1990 (wie Anm. 5), 314.

42 Gerade die methodische Ausrichtung der sogenannten Warburg-Schule wurde zum Paradebeispiel der emigrierten Kunstgeschichte; vgl. Karen Michels, Transplantierte Kunstwissenschaft. Deutschsprachige Kunstgeschichte im amerikanischen Exil (Studien aus dem Warburg-Haus, 2), Berlin 1999, 194.

43 Vgl. Sauerländer 1990 (wie Anm. 5), 315. 
den Sozialwissenschaften der späten 20er Jahre hatte. ${ }^{44}$ Und diese zielten gerade nicht auf selbstvergewissernde Herleitungen oder Rückführungen, sondern auf den spezifischen zeitgenössischen Ausdrucksgehalt der jeweils zu untersuchenden historischen Position.

In den Arbeiten Sauerländers ist der Poussin-Aufsatz in methodischer Hinsicht ein Solitär. Die geplante, daran anknüpfende Habiliationsschrift über das Erhabene im 18. Jahrhundert wurde nicht weiter verfolgt. Vermutlich hat das mit dem sich ändernden Klima in der Kunstgeschichte dieser Jahre zu tun; und vielleicht hängt das auch mit einem wachsenden Bewußtsein dafür zusammen, daß eine kritische Kunstgeschichte nicht allein eine Frage des methodischen Ansatzes, sondern auch eine Frage der intellektuellen Schärfe und Risikobereitschaft in der praktischen Anwendung ist. In jedem Fall signalisiert der Jahreszeiten-Aufsatz das Bedürfnis nach einem methodischen Neuaufbruch. In der Rückführung der Bildaussagen auf Konzepte, die bis ins Mittelalter zurückreichen, traf Sauerländer jedoch unwillkürlich auf ein sich in Westdeutschland herausbildendes Bedürfnis nach Selbstvergewisserung in der Rückorientierung auf die vermeintliche ,Wiege eines geeinten Europas im christlichen Mittelalter, gegen das er sich selbst ausdrücklich immer wieder ausgesprochen hat.

Vielleicht war der beschriebene methodische Ausbruch Sauerländers in bis dahin ungewohnte Gefilde unumgänglich, um eine neue Perspektive auf die mittelalterliche Skulptur zu entwickeln, der er letztlich als einem zentralen Arbeitsgebiet treu blieb. Wie weitreichend sich diese Perspektive in den folgenden Jahrzehnten wandelte und sich mit Überlegungen zum Zustandekommen der jeweiligen wissenschaftlichen Perspektiven verband, dafür mag hier ein einziger bloßer Hinweis genügen: auf die kritischen Überlegungen zum Stand der Skulpturenforschung, die Sauerländer 22 Jahre später, 1978, in der Zeitschrift für Kunstgeschichte veröffentlichte. ${ }^{45}$

Jetzt darf man gespannt sein auf die angekündigte erneute Auseinandersetzung Sauerländers mit den »Vier Jahreszeiten« Poussins nach nunmehr 50 Jahren.

44 Unmittelbar bezieht sich Panofsky auf Karl Mannheim, Beiträge zur Theorie der Weltanschauungs-Interpretation, in: Wiener Jahrbuch für Kunstgeschichte N. F. 1, 1921/1922 (1923 zwischen zwei Aufsätzen Panofskys erschienen); vgl. auch Rudolf Carnap, Der logische Aufbau der Welt, 3. Aufl. Hamburg 1966 (1. Aufl. 1928).

45 Vgl. zum Beispiel Willibald Sauerländer, Spätstaufische Figuren in Sachen und Thüringen. Überlegungen zum Stand der Forschung, in: Zeitschrift für Kunstgeschichte 41, 1978, 181-216. 